\title{
Biomechanical CT metrics are associated with patient outcomes in COPD
}

\author{
Sandeep Bodduluri, ${ }^{1}$ Surya P Bhatt, ${ }^{2,3,4}$ Eric A Hoffman, ${ }^{1,5}$ John D Newell Jr, ${ }^{1,5}$ \\ Carlos H Martinez, ${ }^{6}$ Mark T Dransfield, ${ }^{2,3,4}$ Meilan K Han, ${ }^{6}$ Joseph M Reinhardt, ${ }^{1}$ \\ for the COPDGene Investigators
}

- Additional material is published online only. To view please visit the journal online (http://dx.doi.org/10.1136/ thoraxinl-2016-209544).

'Department of Biomedical Engineering, University of lowa, lowa City, lowa, USA

${ }^{2}$ Division of Pulmonary, Allergy and Critical Care Medicine,

University of Alabama at Birmingham, Birmingham, Alabama, USA

${ }^{3} \mathrm{UAB}$ Lung Health Center, University of Alabama at Birmingham, Birmingham, Alabama, USA

${ }^{4} \mathrm{UAB}$ Lung Imaging Core, University of Alabama at Birmingham, Birmingham, Alabama, USA

${ }^{5}$ Department of Radiology, University of lowa Carver College of Medicine, lowa City, lowa, USA

${ }^{6}$ Division of Pulmonary and Critical Care Medicine, University of Michigan, Ann Arbor, Michigan, USA

\section{Correspondence to}

Dr Surya P Bhatt, Division of Pulmonary, Allergy and Critical Care Medicine, University of Alabama at Birmingham, THT 422, 1720, 2nd Avenue South, Birmingham, AL 35294, USA; sbhatt@uabmc.edu

SB and SPB contributed equally to the manuscript and are cofirst authors.

Received 6 October 2016 Revised 21 November 2016 Accepted 25 November 2016 Published Online First

2 January 2017

\begin{abstract}
Background Traditional metrics of lung disease such as those derived from spirometry and static singlevolume $\mathrm{CT}$ images are used to explain respiratory morbidity in patients with COPD, but are insufficient. We hypothesised that the mean Jacobian determinant, a measure of local lung expansion and contraction with respiration, would contribute independently to clinically relevant functional outcomes.
\end{abstract}

Methods We applied image registration techniques to paired inspiratory-expiratory CT scans and derived the Jacobian determinant of the deformation field between the two lung volumes to map local volume change with respiration. We analysed 490 participants with COPD with multivariable regression models to assess strengths of association between traditional CT metrics of disease and the Jacobian determinant with respiratory morbidity including dyspnoea (modified Medical Research Council), St Georges Respiratory Questionnaire (SGRQ) score, 6-min walk distance (6MWD) and the Body Mass Index, Airflow Obstruction, Dyspnoea and Exercise Capacity (BODE) index, as well as all-cause mortality.

Results The Jacobian determinant was significantly associated with SGRQ (adjusted regression coefficient $\beta=-11.75,95 \% \mathrm{Cl}-21.6$ to $-1.7 ; p=0.020)$, and with 6MWD ( $\beta=321.15,95 \%$ Cl 134.1 to $508.1 ; p<0.001)$, independent of age, sex, race, body mass index, $\mathrm{FEV}_{1}$, smoking pack-years, CT emphysema, CT gas trapping, airway wall thickness and $\mathrm{CT}$ scanner type. The mean Jacobian determinant was also independently associated with the BODE index $(\beta=-0.41,95 \% \mathrm{Cl}-0.80$ to $-0.02 ; p=0.039$ ) and mortality on follow-up (adjusted $H R=4.26,95 \% \mathrm{Cl} 0.93$ to $19.23 ; p=0.064$ ).

Conclusions Biomechanical metrics representing local lung expansion and contraction improve prediction of respiratory morbidity and mortality and offer additional prognostic information beyond traditional measures of lung function and static single-volume CT metrics.

Trial registration number NCT00608764; Post-results.

\section{BACKGROUND}

Airflow obstruction is the hallmark of COPD; CrossMark

To cite: Bodduluri $S$

Bhatt SP, Hoffman EA, et al.

Thorax 2017;72:409-414. however, CT is increasingly used to characterise and phenotype subtypes of COPD. ${ }^{1}$ The major subtypes of structural lung disease visualised on CT, emphysema and thickened airway walls, are both independently associated with airflow obstruction. ${ }^{2}{ }^{3}$ These metrics of disease on CT have also

\section{Key messages}

What is the key question?

- Traditional metrics of lung disease such as spirometry and static single-volume CT images are used to explain respiratory morbidity in patients with COPD. However, there is substantial discordance in individual patients between these metrics and respiratory morbidity. Can biomechanical measures of local lung expansion and contraction offer additional prognostic information in COPD?

\section{What is the bottom line?}

- We demonstrate that biomechanical metrics of local lung expansion and contraction offer additional information beyond traditional measures of lung function and static single-volume CT metrics in the prediction of respiratory morbidity and all-cause mortality.

\section{Why read on?}

- Independent prognostic information offered by biomechanical measures will help improve prognostication and phenotyping patients with COPD.

been shown to be associated with important patient-reported and objective outcomes such as dyspnoea, ${ }^{4}$ quality of life, ${ }^{5}{ }^{6}$ exercise capacity, ${ }^{78}$ the Body Mass Index, Airflow Obstruction, Dyspnoea and Exercise Capacity (BODE) index ${ }^{5}$ and mortality. ${ }^{9}$ A substantial number of patients with COPD, however, have significant discordance between spirometry and CT findings. We recently demonstrated that a biomechanical measure of structural lung disease (the Jacobian determinant, a measure of local lung volume change assessed through matching images acquired at multiple lung volumes) substantially explains the differences between static single-volume CT images and airflow obstruction on spirometry. ${ }^{10}{ }^{11}$ However, it remains important to determine whether the Jacobian determinant is associated with standard clinical measures of patient outcomes. Such determination will enhance its use in better phenotyping patients. We analysed data from a large subset of a well-characterised cohort of participants with 
COPD who underwent clinical, physiological and radiographic assessments. We hypothesised that the mean Jacobian determinant, would provide additional explanation for the variations in clinically relevant outcomes including dyspnoea, quality of life, functional capacity and the BODE index, as well as all-cause mortality.

\section{METHODS}

\section{Patient selection}

We analysed participants enrolled in the Genetic Epidemiology of COPD (COPDGene) study, a large, ongoing, multicentre cohort study that includes current and former smokers aged 4580 years and without other chronic lung diseases except COPD and asthma. Demographics were collected as per the COPDGene protocol via self-administered questionnaires. Details of the study protocol have been previously published. ${ }^{12}$ Post-bronchodilator spirometry was performed using the ndd EasyOne spirometer to assess airflow obstruction. COPD was diagnosed using the ratio of $\mathrm{FEV}_{1}$ to FVC of $<0.70$, and severity categorised according to Global initiative for Chronic obstructive Lung Disease (GOLD) recommendations. ${ }^{13}$ All participants provided written informed consent and the research protocol was approved by the institutional review board at each participating centre. The first 1000 participants to enrol, and with complete image registration CT data, were included in the study reported here.

\section{Morbidity data}

Respiratory-related quality of life was assessed using the St. George's Respiratory Questionnaire (SGRQ). ${ }^{14}$ Scores were calculated for the three main subdomains of the SGRQ: symptoms, activities and impact. Total score ranges from 0 to 100 , with higher scores indicating worse quality of life. The minimum clinically important difference (MCID) for SGRQ is 4 units. Dyspnoea was quantified using the modified Medical Research Council (mMRC) dyspnoea score; the score ranges from 0 to 4 , with greater score indicating worse dyspnoea. ${ }^{15}$ Exercise tolerance was assessed using the $6 \mathrm{~min}$ walk distance (6MWD); MCID for the 6MWD is $26 \mathrm{~m} .{ }^{16}$ The multidimensional BODE index was finally calculated using body mass index (BMI), $\mathrm{FEV}_{1} \%$ predicted, $\mathrm{mMRC}$ and $6 \mathrm{MWD}$; the scale ranges from 0 to 10 , with greater scores indicating a higher risk of mortality. ${ }^{17}$ We also obtained data on all-cause mortality on longitudinal follow-up of approximately 5 years.

\section{CT image acquisition}

Volumetric CT scans were acquired with the subject in the supine position during a carefully coached breath-hold to either full inspiration (total lung capacity (TLC)) or end expiration (functional residual capacity (FRC)). The scanning protocol included a collimation, $0.5 \mathrm{~mm}$; tube voltage, $120 \mathrm{kV}$; tube current, $200 \mathrm{mAs}$; gantry rotation time, $0.5 \mathrm{~s}$; and a pitch, 1.1. The images were reconstructed with a standard kernel (dependent upon the make and model of the scanner) and a slice thickness of $0.75 \mathrm{~mm}$ and an interval of $0.5 \mathrm{~mm}$.

\section{CT image-based measures \\ Densitometry}

Using 3D Slicer software (http://www.airwayinspector.org), we measured emphysema and gas trapping based on density mask analyses. ${ }^{12}$ Emphysema was quantified by using the percentage of voxels at TLC with attenuation <-950 Hounsfield units (HU) and gas trapping was quantified as the low attenuation areas on FRC scan that are $<-856$ HU. We used Pulmonary
Workstation 2 (VIDA Diagnostics, Coralville, Iowa, USA) to measure the wall area percentage of segmental airways (WA\%) that represent airway remodelling. ${ }^{12}$ The WA\% was quantified at full-inspiration CT image and was estimated as (wall area/ total bronchial area) $\times 100$. The mean WA $\%$ was calculated as the average of six segmental bronchi in each subject.

Image registration

The TLC and FRC scans were registered for each subject. Details of image registration are provided in the online supplementary material. Briefly, a lung mass-preserving registration method was used to capture volume changes between the two phases of respiration. ${ }^{18}$ A sum of squared tissue volume difference method was used as a similarity metric. This method has been shown previously to be effective in lung image registration protocols. ${ }^{10} 11$ The transformation matrix from the registration process was used to derive regional tissue expansion and contraction measures between TLC and FRC volumes. In this study, we used the Jacobian determinant metric to represent regional deformation patterns in patients with COPD. The Jacobian determinant measures the local volume change and estimates the pointwise expansion and contraction during deformation of the lung from TLC to FRC. This results in a deformation map that has values ranging from 0 to infinity. A Jacobian determinant value $>1$ indicates local expansion, whereas $<1$ indicates local contraction. A Jacobian determinant value equal to 1 indicates neither local expansion nor contraction. We used the mean of the Jacobian deformation map as a lung mechanical measure for each subject representing local deformation patterns between TLC and FRC volumes. Figure 1 shows representative Jacobian deformation maps for participants with different disease severity.

\section{Statistical analyses}

We expressed all values as mean (SD). Pearson and Spearman correlation analyses were performed to assess the pairwise relationship of Jacobian determinant with SGRQ scores, BODE index, mMRC, 6MWD and $\mathrm{FEV}_{1}$. Association between Jacobian determinant and SGRQ was assessed using univariate and multivariable linear regression models after adjustment for age, sex, race, pack-years, BMI, FEV ${ }_{1}$, CT emphysema, CT gas trapping, WA $\%$ and CT scanner type. As there was an overrepresentation of zero BODE scores, we used zero-inflated Poisson regression analyses to test the association between Jacobian determinant and the BODE index, after adjustment for the above-mentioned variables, except for $\mathrm{FEV}_{1}$ and BMI as they are part of the BODE index. To calculate prediction of mortality on follow-up, we performed Cox proportional hazards analysis. Variables significant on univariate analysis at $\mathrm{p}<0.05$ were entered into a multivariable Cox proportional hazards model to calculate adjusted HR for mortality for the Jacobian determinant; we forced CT scanner type into the model as this is clinically important. All tests of significance were two-tailed, and we considered an $\alpha$ level of $<0.05$ as statistically significant. All analyses were performed using Statistical Package for the Social Sciences (SPSS V.22.0, SPSS, Chicago, Illinois, USA) and R statistical software (V.3.2).

\section{RESULTS}

\section{Demographics}

Of the first 1000 participants to enrol, 562 participants had COPD GOLD stages $1-4$. We excluded 72 participants due to image registration errors in boundary alignment. Table 1 shows the baseline demographics, physiologic assessments, CT 
Figure $1 \quad(A, C)$ Axial $C T$ section and respectively for a representative participant with Global initiative for Chronic obstructive Lung Disease (GOLD) 1 with low Body Mass Index, Airflow Obstruction, Dyspnoea and Exercise Capacity (BODE) index. (C, D) Similar images for a representative participant with GOLD 4 COPD and high BODE index. The colours depict the Jacobian deformation map from full inspiration to end expiration and show the variability in regional tissue expansion patterns across both subjects. Jacobian determinant $=1$ represents no deformation; $>1=$ local expansion; and $<1=$ local contraction. the corresponding deformation map

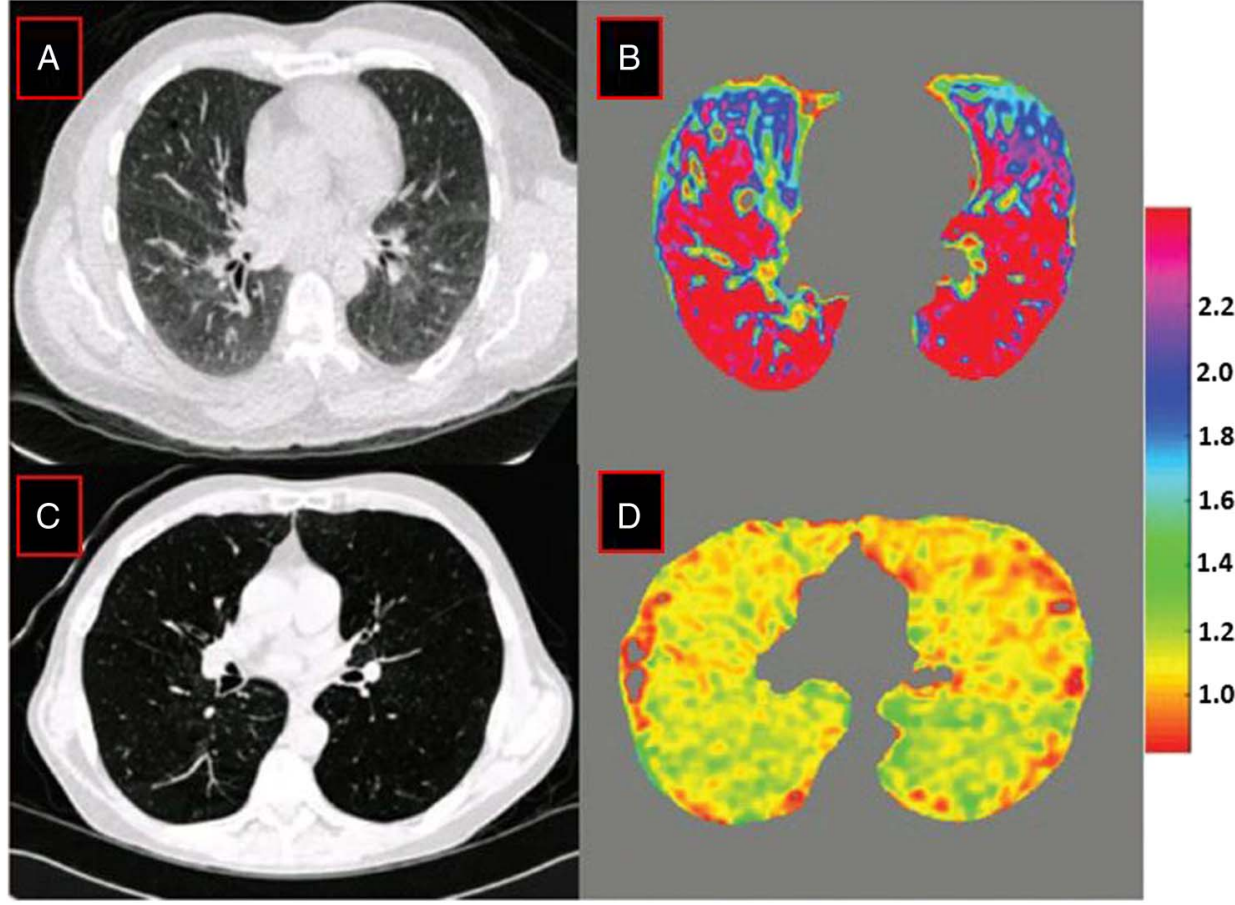

Table 1 Baseline demographics, physiological assessments, CT quantification of COPD and morbidity scores

\begin{tabular}{lc}
\hline Parameter & Mean (SD) \\
\hline Age (years) & $64.6(8.2)$ \\
Female (\%) & $240(49)$ \\
African-American (\%) & $78(16)$ \\
BMI (kg/m²) & $27.5(5.8)$ \\
Smoking pack-years & $52.9(26.1)$ \\
Current smokers (\%) & $336(68)$ \\
FEV 1 (L) & $1.49(0.7)$ \\
FEV ${ }_{1} \%$ predicted & $53.2(22.0)$ \\
FVC (L) & $2.96(1.0)$ \\
FVC \% predicted & $80.0(21.3)$ \\
FEV 1 /FVC & $0.49(0.12)$ \\
CT emphysema (\%) & $12.4(12.1)$ \\
CT gas trapping (\%) & $37.9(20.3)$ \\
Airway wall area (\%), segmental airways & $62.3(3.1)$ \\
SGRQ (total score) & $37.6(22.4)$ \\
6MWD (m) & $360(145)$ \\
BODE index* & $3(1-5)$ \\
mMRC score* & $2(1-3)$ \\
\hline All values expressed as mean (SD) or as percentage, except * where median (IQR) are \\
depicted. & \\
6 MWD, 6 min walk distance; BMI, body mass index; BODE index, BMI, Airflow \\
Obstruction, Dyspnoea and Exercise capacity; mMRC, modified Medical Research \\
Council dyspnoea scale; SGRQ, St George's Respiratory Questionnaire. & \\
\hline &
\end{tabular}

quantification of COPD and respiratory morbidity scores. The mean age of the participants was 64.6 (8.2) years. The cohort was comprised of 250 (51.1\%) males and 240 (48.9\%) non-Hispanic whites. Participants had a substantial cigarette smoking burden with mean pack-years of 52.9 (26.1); 336 $(68 \%)$ were active smokers at the time of enrolment. The participants encompassed the spectrum of disease severity, with 62 (12.7\%), 204 (41.6\%), 138 (28.2\%) and 86 (17.6\%) having GOLD severity stages 1 through 4, respectively. Distributions of
SGRQ, 6MWD and the BODE index in the study population are shown in online supplementary figure S2.

\section{Correlation between the Jacobian measure and respiratory morbidity}

Pairwise relationship analysis between the Jacobian determinant and patient outcomes was performed. There was an inverse and statistically significant association between the Jacobian determinant and total SGRQ scores $(\mathrm{r}=-0.46 ; \mathrm{p}<0.001)$, the BODE index $(\mathrm{r}=-0.60 ; \mathrm{p}<0.001)$ and mMRC scores $(\mathrm{r}=-0.41$; $\mathrm{p}<0.001)$. There was a positive association between the Jacobian determinant and the 6MWD $(r=0.47 ; \mathrm{p}<0.001)$ and $\mathrm{FEV}_{1}(\mathrm{r}=0.60 ; \mathrm{p}<0.001)$.

\section{CT measures and SGRQ}

Table 2 shows univariate and multivariable associations between the CT metrics of emphysema, gas trapping, WA\% and the Jacobian measure with SGRQ. On univariate regression, the Jacobian determinant was significantly associated with SGRQ (unadjusted regression coefficient $\beta=-38.1,95 \%$ CI -46.8 to -29.2; $\mathrm{p}<0.001)$; this relationship held true after adjustment for age, sex, race, BMI, $\mathrm{FEV}_{1}$, smoking pack-years, CT emphysema, CT gas trapping, airway wall area (WA\%) and CT scanner type $(\beta=-11.75,95 \% \mathrm{CI}-21.6$ to $-1.7 ; \mathrm{p}=0.020)$.

\section{CT measures and 6MWD}

The relationship between CT metrics and the 6MWD is shown in table 3. On univariate analysis, the Jacobian measure was significantly associated with distance walked $(\beta=770.1,95 \% \mathrm{CI}$ 600.0 to $940.1 ; \mathrm{p}<0.001)$; this association was maintained after multivariable adjustment $(\beta=321.15$, 95\% CI 134.1 to 508.1; $\mathrm{p}<0.001)$.

\section{CT measures and BODE index}

Table 4 shows the relationships between CT metrics and BODE index. The Jacobian determinant was significantly associated with the BODE index on univariate analysis $(\beta=-1.77,95 \% \mathrm{CI}$ -2.04 to $-1.51 ; \mathrm{p}<0.001)$, and after adjustment for age, sex, 
Table 2 Univariate and multivariable associations of mean Jacobian determinant measure with SGRQ

\begin{tabular}{|c|c|c|c|c|}
\hline \multirow[b]{2}{*}{ Parameter } & \multicolumn{2}{|l|}{ Univariate regression } & \multicolumn{2}{|l|}{ Multivariable regression } \\
\hline & Regression coefficient $\boldsymbol{\beta}(95 \% \mathrm{Cl})$ & $p$ Value & Regression coefficient $\boldsymbol{\beta}(95 \% \mathrm{Cl})$ & $p$ Value \\
\hline Mean Jacobian determinant & $-38.08(-46.8$ to -29.2$)$ & $<0.001$ & $-11.75(-21.6$ to -1.7$)$ & 0.020 \\
\hline Age (years) & $-0.50(-0.7$ to -0.2$)$ & $<0.001$ & $-0.68(-0.9$ to -0.4$)$ & $<0.001$ \\
\hline African-American race & $12.22(6.9$ to 17.5$)$ & $<0.001$ & 6.73 (1.6 to 11.7$)$ & 0.009 \\
\hline Female sex & $-0.91(-4.9$ to 3.0$)$ & 0.652 & $-1.69(-5.5$ to 2.1$)$ & 0.390 \\
\hline BMI $\left(\mathrm{kg} / \mathrm{m}^{2}\right)$ & $0.21(-0.1$ to 0.5$)$ & 0.219 & $0.39(0.0$ to 0.7$)$ & 0.013 \\
\hline $\mathrm{FEV}_{1}(\mathrm{~L})$ & $-15.4(-17.8$ to -13.2$)$ & $<0.001$ & $-10.65(-14.2$ to -7.0$)$ & $<0.001$ \\
\hline Smoking pack-years & 0.11 (0.03 to 0.18$)$ & 0.003 & $0.16(0.1$ to 0.2$)$ & $<0.001$ \\
\hline CT emphysema (\%) & $0.57(0.4$ to 0.7$)$ & $<0.001$ & $0.33(0.0$ to 0.5$)$ & 0.002 \\
\hline CT gas trapping (\%) & $0.44(0.3$ to 0.5$)$ & $<0.001$ & $-0.01(-0.1$ to 0.1$)$ & 0.990 \\
\hline Airway wall area (\%) & 2.51 (1.8 to 3.1$)$ & $<0.001$ & 0.95 (0.3 to 1.5$)$ & 0.002 \\
\hline
\end{tabular}

Multivariable regression model adjusted for CT scanner type.

BMI, body mass index; SGRQ, St George's Respiratory Questionnaire.

race, smoking pack-years, CT emphysema, CT gas trapping, airway wall area per cent and CT scanner type $(\beta=-0.4,95 \%$ CI -0.8 to $-0.01 ; \mathrm{p}=0.039)$.

\section{CT measures and mortality}

We had follow-up data for 441 of the 490 participants for a median of 6.8 years (IQR 6.6-6.9). Of these, 99 (22.4\%) died on follow-up. On univariate analyses, all CT measures were associated with all-cause mortality; unadjusted HR for CT emphysema 1.03 (95\% CI 1.02 to $1.05 ; \mathrm{p}<0.001)$, CT gas trapping 1.03 (95\% CI 1.02 to $1.04 ; \mathrm{p}<0.001)$, WA\% $1.08(95 \%$ CI 1.01 to $1.15 ; \mathrm{p}<0.001)$ and lower Jacobian determinant 13.16 (95\% CI 4.52 to 38.46 ; $\mathrm{p}<0.001)$. After adjustment for age, pack-years of smoking, $\mathrm{FEV}_{1}$, CT gas trapping, WA\% and CT scanner type, CT emphysema was not associated with mortality (adjusted HR 1.01, 95\% CI 0.98 to $1.04 ; \mathrm{p}=0.446$ ). WA $\%$ and CT gas trapping were also not independently associated with mortality (adjusted HR 1.05, 95\% CI 0.96 to 1.14; $\mathrm{p}=0.315$; and $1.01,95 \%$ CI 0.98 to $1.04 ; \mathrm{p}=0.424$, respectively). After adjustment for age, pack-years of smoking, $\mathrm{FEV}_{1}$, CT emphysema, CT gas trapping, WA\% and CT scanner type, a lower Jacobian determinant approached statistical significance for association with mortality (adjusted HR 4.26, 95\% CI 0.93 to $19.23 ; \mathrm{p}=0.064$ ).

\section{DISCUSSION}

We demonstrated a strong relationship between the Jacobian determinant mean, a biomechanical measure of regional parenchymal volume change, and important patient outcomes including dyspnoea, respiratory quality of life, functional capacity and the BODE index, and mortality. On multivariable analyses, we found that the effect size of a unit change in the Jacobian determinant is stronger than the effect sizes of static single-volume CT measures of COPD such as emphysema percentage, gas trapping and airway wall thickness, thus providing novel and independent information applicable to COPD phenotyping and prognosis.

It is well established that there is, at best, a modest correlation between $\mathrm{FEV}_{1}$, the primary measure of disease severity in COPD, and clinical outcomes such as respiratory health-related quality of life and dyspnoea, ${ }^{19}{ }^{20}$ and $\mathrm{FEV}_{1}$ does not fully explain the morbidity associated with COPD. In this regard, our findings extend the results of other recent studies that demonstrated a relationship between CT metrics of COPD and clinically relevant outcomes. Although lung function impairment is correlated with poor functional outcomes, SGRQ is influenced by a number of factors that are not fully explained by $\mathrm{FEV}_{1}$ such as cough and exacerbations. Grydeland and colleagues demonstrated that CT indices of emphysema and airway disease improved the prediction of respiratory symptoms over spirometry alone. ${ }^{4}$ Gietema et $a l^{6}$ showed that both airway wall

Table 3 Univariate and multivariate association of mean Jacobian determinant measure with 6 min distance walked

\begin{tabular}{|c|c|c|c|c|}
\hline \multirow[b]{2}{*}{ Parameter } & \multicolumn{2}{|l|}{ Univariate regression } & \multicolumn{2}{|l|}{ Multivariable regression } \\
\hline & Regression coefficient $\boldsymbol{\beta}(95 \% \mathrm{Cl})$ & $\mathrm{p}$ Value & Regression coefficient $\boldsymbol{\beta}(95 \% \mathrm{Cl})$ & $\mathrm{p}$ Value \\
\hline Mean Jacobian determinant & $770.10(600.0$ to 940.1$)$ & $<0.001$ & 321.15 (134.1 to 508.1$)$ & $<0.001$ \\
\hline Age (years) & $-2.75(-7.4$ to 1.9$)$ & 0.251 & $-3.52(-7.7$ to 0.6$)$ & 0.097 \\
\hline African-American race & $-331.10(-431.6$ to -230.5$)$ & $<0.001$ & $-290.51(-385.6$ to -195.3$)$ & $<0.001$ \\
\hline Female sex & $-74.66(-151.8$ to 2.49$)$ & 0.057 & $-31.00(-103.6$ to 41.6$)$ & 0.402 \\
\hline Body mass index $\left(\mathrm{kg} / \mathrm{m}^{2}\right)$ & $-7.07(-13.6$ to -0.5$)$ & 0.035 & $-10.79(-16.6$ to 41.6$)$ & $<0.001$ \\
\hline $\mathrm{FEV}_{1}(\mathrm{~L})$ & 315.72 (270.7 to 360.6$)$ & $<0.001$ & 204.08 (136.2 to 271.9$)$ & $<0.001$ \\
\hline Smoking pack-years & $-1.18(-2.6$ to 0.2$)$ & 0.115 & $-2.14(-3.3$ to -0.9$)$ & $<0.001$ \\
\hline CT emphysema (\%) & $-8.19(-11.3$ to -5.0$)$ & $<0.001$ & $-7.26(-11.8$ to -2.6$)$ & 0.001 \\
\hline CT gas trapping (\%) & $-7.10(-9.0$ to -5.1$)$ & $<0.001$ & $2.95(-0.3$ to 6.2$)$ & 0.081 \\
\hline Airway wall area (\%) & $-40.24(-52.3$ to -28.2$)$ & $<0.001$ & $-12.03(-23.5$ to -0.5$)$ & 0.040 \\
\hline
\end{tabular}


Table 4 Univariate and multivariable associations of mean Jacobian determinant measure with Body Mass Index, Airflow Obstruction, Dyspnoea and Exercise capacity index

\begin{tabular}{|c|c|c|c|c|}
\hline \multirow[b]{2}{*}{ Parameter } & \multicolumn{2}{|l|}{ Univariate regression } & \multicolumn{2}{|l|}{ Multivariable regression } \\
\hline & Regression coefficient $\boldsymbol{\beta}(95 \% \mathrm{CI})$ & p Value & Regression coefficient $\boldsymbol{\beta}(95 \% \mathrm{CI})$ & p Value \\
\hline Mean Jacobian determinant & $-1.77(-2.044$ to -1.507$)$ & $<0.001$ & $-0.41(-0.803$ to -0.019$)$ & 0.039 \\
\hline Age (years) & $-0.004(-0.010$ to 0.002$)$ & 0.189 & $-0.04(-0.014$ to 0.001$)$ & 0.054 \\
\hline African-American race & $0.22(0.083$ to 0.350$)$ & 0.001 & $0.16(-0.008$ to 0.325$)$ & 0.059 \\
\hline Female sex & $-0.01(-0.112$ to 0.082$)$ & 0.783 & $0.15(0.040$ to 0.275$)$ & 0.008 \\
\hline Smoking pack-years & $0.002(0.003$ to 0.004$)$ & 0.013 & $0.00(0.001$ to 0.005$)$ & 0.001 \\
\hline CT emphysema (\%) & $0.02(0.021$ to 0.029$)$ & $<0.001$ & $0.01(0.003$ to 0.018$)$ & 0.003 \\
\hline CT gas trapping (\%) & $0.02(0.023$ to 0.028$)$ & $<0.001$ & $0.01(0.009$ to 0.020$)$ & $<0.001$ \\
\hline Airway wall area (\%) & $0.08(0.064$ to 0.102$)$ & $<0.001$ & $0.07(0.050$ to 0.089$)$ & $<0.001$ \\
\hline
\end{tabular}

thickness and emphysema are independently associated with respiratory quality of life, and Martinez et $a l^{5}$ reported that there was a stronger association between SGRQ and airway wall thickness than between SGRQ and CT emphysema. On the other hand, they also found that the BODE index was influenced more by emphysema than by airway wall thickness. Other studies have also found significant associations between measures of emphysema and airflow obstruction, exercise tolerance and dyspnoea. 237821

The possible reasons for why the Jacobian determinant shows independent associations with outcomes merit discussion. $\mathrm{FEV}_{1}$ is a global measure of lung function impairment and is influenced by both airway narrowing and decreased lung elastic recoil associated with emphysema. However, in a given patient, it is usual to see multiple subtypes of emphysema such as centrilobular and panlobular emphysema with differential impact on lung function. ${ }^{22-24}$ The degree of CT emphysema does not translate linearly into airflow obstruction, ${ }^{3} 10$ and it is likely that different types and distribution patterns of emphysema contribute differentially to airflow obstruction. ${ }^{22-24}$ Although we did not study emphysema subtypes, the mean Jacobian determinant, by offering information of regional volume change with respiration, likely reflects a more direct physiological link between structural lung disease and the lung mechanics reflected by spirometry. Previous studies have found that imaging metrics offered marginal additional, albeit independent information, over that offered by spirometry in the prediction of respiratory quality of life. However, we showed that CT-based metrics derived from image-matching offer information that are not only independent and additive, but with effect sizes greater than $\mathrm{FEV}_{1}$ for all the outcomes studied. In addition, dyspnoea contributes significantly to respiratory quality of life as well as reduction in functional capacity, and even patients with relatively preserved $\mathrm{FEV}_{1}$ can experience significant dyspnoea and hence a poorer quality of life. This could be due to factors other than $\mathrm{FEV}_{1}$ such as poorer diaphragmatic position due to resting as well as dynamic hyperinflation, both of which might be reflected better by changes in regional volume change than by static single-volume CT measures of emphysema and airway disease. Our findings provide new insight into the complex pathophysiological basis and the heterogeneity of causes of changes in dyspnoea and SGRQ. These findings are pertinent as dyspnoea and SGRQ are frequently used as outcome measures in clinical trials of pharmacologic and non-pharmacological therapies, once again highlighting the importance of phenotyping patients with COPD.
We also showed that the Jacobian estimate of local lung volume change was significantly associated with the BODE index that predicts all-cause and respiratory-specific mortality in COPD. ${ }^{17}$ The association between mean Jacobian determinant and mortality approached statistical significance. This is a novel finding and adds to the findings of previous studies, which demonstrated a relationship between CT emphysema and both the BODE index and mortality. ${ }^{5} 925$ Our findings are aligned with those of Martinez et al, ${ }^{26}$ who found that CT emphysema was not associated with mortality, but are in contrast to those of Johannessen et al, ${ }^{9}$ who found that patients with more severe emphysema have higher mortality compared with those with mild emphysema. It is unclear whether the poorer mechanics are a more sensitive reflector of underlying emphysema that has been shown to be independently associated with poor outcomes and mortality, ${ }^{27}$ or if the affected lung mechanics have an independent role in disease progression and mortality. Of note, lung volume reduction surgery improves symptoms and mortality in a subset of patients, ${ }^{28}$ and it is plausible that the benefits of lung volume reduction are largely due to improved lung elasticity in the ipsilateral preserved lobe. ${ }^{29}$

One of the limitations of our study is that the CT scans were not spirometrically controlled. ${ }^{3031}$ As varying respiratory effort can affect the reproducibility of image-registration metrics, participants were coached to maximum inhalation and end expiration. As this was a multicentre trial, a number of different scanners were used for image acquisition; however, we adjusted for scanner type. For calculating the Jacobian determinant, CT scans were obtained at only two volumes. Although this limited our ability to assess regional differences in lung mechanics along the entire spectrum of lung inflation and deflation, ${ }^{32}$ our metrics are applicable to clinically obtained scans without the excessive radiation risks that would be incurred in acquiring dynamic scans. We did not have mortality data in some participants, and this could have introduced some selection bias; however, we had survival data on most participants. Our study was strengthened by the inclusion of participants enrolled in a large well-characterised cohort of current and former smokers that included a high proportion of African-Americans.

In conclusion, biomechanical metrics of local lung expansion and contraction offer better prediction of respiratory quality of life, the BODE index and mortality, and offer additional prognostic information beyond traditional measures of lung function and static single-volume CT metrics. Traditional CT measures have been used to phenotype COPD, and the implications of these phenotyping metrics have been assessed by correlating 
with relevant clinical outcomes. Thus, the Jacobian determinant, by offering additional information on lung disease in COPD, and by showing additional associations with clinically relevant outcomes, can add to our ability to phenotype patients with COPD based on the complex pathophysiological heterogeneity that extends beyond lung function measures alone.

\section{Collaborators COPDGene Investigators.}

Contributors SB and SPB contributed equally to the article. Study concept and design: SPB, SB and JMR. Acquisition, analysis or interpretation of data, critical revision of the manuscript for important intellectual content and study supervision: all authors. Drafting of the manuscript and statistical analysis: SB and SPB.

Funding This study was supported by the COPDGene study (NIH Grant Numbers R01 HL089897 and R01 HL089856), 1 K23HL133438-01 (SPB), R01 HL112986 (EAH) and R01 HL079406 (JMR).

Competing interests SPB reports grants from the NIH; MTD reports grants from $\mathrm{NHLBI}$, during the conduct of the study; personal fees and other from Boehringer Ingelheim, personal fees and other from AstraZeneca, personal fees and other from GlaxoSmithKline, personal fees from Genentech, other from Novartis, other from PneumRx, other from Pulmonx, other from Yungjin; EAH is a founder and share holder of VIDA Diagnostics, a company that is commercializing lung image analysis software developed, in part, at the University of lowa; CHM reports grants from $\mathrm{NIH}$; MKH reports personal fees from $\mathrm{BI}$, personal fees from GSK, personal fees from AstraZeneca, personal fees from Novartis; JMR reports other from VIDA Diagnostics; JDN reports grants from $\mathrm{NIH}$, during the conduct of the study; grants from $\mathrm{NIH}$, grants from Siemens Healthcare, personal fees from VIDA Diagnostics, personal fees from GSK.

Ethics approval Institutional review board at all 21 participating centres.

Provenance and peer review Not commissioned; externally peer reviewed.

\section{REFERENCES}

1 Han MK. Clinical correlations of computed tomography imaging in chronic obstructive pulmonary disease. Ann Am Thorac Soc 2013;10(Suppl):S131-7.

2 Schroeder JD, McKenzie AS, Zach JA, et al. Relationships between airflow obstruction and quantitative CT measurements of emphysema, air trapping, and airways in subjects with and without chronic obstructive pulmonary disease. AJR Am J Roentgenol 2013;201:W460-70.

3 Bhatt SP, Sieren JC, Dransfield MT, et al. Comparison of spirometric thresholds in diagnosing smoking-related airflow obstruction. Thorax 2014;69:409-14.

4 Grydeland TB, Dirksen A, Coxson HO, et al. Quantitative computed tomography measures of emphysema and airway wall thickness are related to respiratory symptoms. Am J Respir Crit Care Med 2010;181:353-9.

5 Martinez $\mathrm{CH}$, Chen YH, Westgate PM, et al. Relationship between quantitative CT metrics and health status and BODE in chronic obstructive pulmonary disease. Thorax 2012;67:399-406.

6 Gietema HA, Edwards LD, Coxson HO, et al. Impact of emphysema and airway wall thickness on quality of life in smoking-related COPD. Respir Med 2013;107:1201-9.

7 Diaz AA, Bartholmai B, San José Estépar R, et al. Relationship of emphysema and airway disease assessed by CT to exercise capacity in COPD. Respir Med 2010;104:1145-51.

8 Spruit MA, Watkins ML, Edwards LD, et al. Determinants of poor 6-min walking distance in patients with COPD: the ECLIPSE cohort. Respir Med 2010;104:849-57.

9 Johannessen A, Skorge TD, Bottai M, et al. Mortality by level of emphysema and airway wall thickness. Am J Respir Crit Care Med 2013;187:602-8.

10 Bhatt SP, Bodduluri S, Newell JD, et al. CT-derived biomechanical metrics improve agreement between spirometry and emphysema. Acad Radiol 2016;23:1255-63.
11 Bodduluri S, Newell JD Jr, Hoffman EA, et al. Registration-based lung mechanical analysis of chronic obstructive pulmonary disease (COPD) using a supervised machine learning framework. Acad Radiol 2013;20:527-36.

12 Regan EA, Hokanson JE, Murphy JR, et al. Genetic epidemiology of COPD (COPDGene) study design. Copd 2010;7:32-43.

13 Vestbo J, Hurd SS, Agusti AG, et al. Global strategy for the diagnosis, management and prevention of chronic obstructive pulmonary disease: GOLD executive summary. Am J Respir Crit Care Med 2013;187:347-65.

14 Jones PW, Quirk FH, Baveystock CM. The St George's respiratory questionnaire. Respir Med 1991;85(Suppl B):25-31; discussion 33-7.

15 Mahler DA, Wells CK. Evaluation of clinical methods for rating dyspnea. Chest 1988;93:580-6.

16 Polkey MI, Spruit MA, Edwards LD, et al. Six-minute-walk test in chronic obstructive pulmonary disease: minimal clinically important difference for death or hospitalization. Am J Respir Crit Care Med 2013;187:382-6.

17 Celli BR, Cote CG, Marin JM, et al. The body-mass index, airflow obstruction, dyspnea, and exercise capacity index in chronic obstructive pulmonary disease. N Engl J Med 2004;350:1005-12.

18 Ding K, Cao K, Fuld MK, et al. Comparison of image registration based measures of regional lung ventilation from dynamic spiral CT with Xe-CT. Med Phys 2012:39:5084-98.

19 Westwood M, Bourbeau J, Jones PW, et al. Relationship between FEV1 change and patient-reported outcomes in randomised trials of inhaled bronchodilators for stable COPD: a systematic review. Respir Res 2011;12:40.

20 Oga T, Nishimura K, Tsukino M, et al. Longitudinal deteriorations in patient reported outcomes in patients with COPD. Respir Med 2007; 101:146-53.

21 Castaldi PJ, San Jose Estepar R, Mendoza CS, et al. Distinct quantitative computed tomography emphysema patterns are associated with physiology and function in smokers. Am J Respir Crit Care Med 2013;188:1083-90.

22 Kim WD, Eidelman DH, Izquierdo JL, et al. Centrilobular and panlobular emphysema in smokers. Two distinct morphologic and functional entities. Am Rev Respir Dis 1991;144:1385-90.

23 Saetta M, Kim WD, Izquierdo JL, et al. Extent of centrilobular and panacinar emphysema in smokers' lungs: pathological and mechanical implications. Eur Respir J 1994;7:664-71.

24 Sverzellati N, Lynch DA, Pistolesi M, et al. Physiologic and quantitative computed tomography differences between centrilobular and panlobular emphysema in COPD. Chronic Obstr Pulm Dis 2014;1:125-32.

25 Haruna A, Muro S, Nakano Y, et al. CT scan findings of emphysema predict mortality in COPD. Chest 2010;138:635-40.

26 Martinez FJ, Foster G, Curtis JL, et al. Predictors of mortality in patients with emphysema and severe airflow obstruction. Am J Respir Crit Care Med 2006:173:1326-34

27 Oelsner EC, Hoffman EA, Folsom AR, et al. Association between emphysema-like lung on cardiac computed tomography and mortality in persons without airflow obstruction: a cohort study. Ann Intern Med 2014;161:863-73.

28 Fishman A, Martinez F, Naunheim K, et al. A randomized trial comparing lung-volume-reduction surgery with medical therapy for severe emphysema. $N$ Engl J Med 2003:348:2059-73.

29 Scharf SM, Rossoff L, McKeon K, et al. Changes in pulmonary mechanics after lung volume reduction surgery. Lung 1998;176:191-204.

30 Fuld MK, Grout RW, Guo J, et al. Systems for lung volume standardization during static and dynamic MDCT-based quantitative assessment of pulmonary structure and function. Acad Radiol 2012;19:930-40.

31 Iyer KS, Grout RW, Zamba GK, et al. Repeatability and sample size assessment associated with computed tomography-based lung density metrics. Chronic Obstr Pulm Dis 2014;1:97-104.

32 Jahani N, Yin Y, Hoffman EA, et al. Assessment of regional non-linear tissue deformation and air volume change of human lungs via image registration. J Biomech 2014;47:1626-33. 